\title{
Opportunity Constraint Evaluation Method for Bottom Stability of Foundation Pit
}

\author{
Xiao-wei Wang ${ }^{1, ~ a ~, ~ X i a o-r u i ~ W a n g ~}{ }^{2, b}$ and Hong-jian Jiang ${ }^{3, c}$ \\ ${ }^{1}$ Luoyang Huitong International Urban Community and 15 buildings, 471000, China \\ ${ }^{2}$ No. 36 North Ring Road, Zhengzhou,450045,China (Corresponding Author) \\ ${ }^{3}$ No. 36 North Ring Road, Zhengzhou,450045,China \\ a1027506898@qq.com, b191847763@qq.com, c1592171001@qq.com
}

\begin{abstract}
Keywords: bottom stability of foundation pit, limit equilibrium theory, random variable, chance-constrained programming.

Abstract. The limit equilibrium theory is employed to analyze the bottom stability of foundation pit, a method that is used to calculate stability coefficient of resisting upheaval in accordance with deterministic mass property and sliding mode. The deterministic model for the analysis of bottom stability that can adapt to different types of slip surface is established by applying the motion-unit method to limit equilibrium theory and taking into account the strength reduction principle. Given the randomness of soil mechanical properties at the pit bottom and the failure of intensity parameter to meet the randomness of constraint conditions, the reliability analysis method for chance constraint is set up. The calculation and comparison between circular sliding surface and Prandtl's sliding surface offers an analysis of the influence of circle center position of different sliding surfaces, load of pit bottom and bracing load on the insertion ratio of supporting structure and the control of confidence level of random constraint function on insertion ratio. The above-mentioned analysis thoughts and methods are achieved through an engineering example
\end{abstract}

\section{Introduction}

While heavily determining the safety, deformation and displacement of supporting structure, the insertion length of supporting structure is also in direct relation to the cost control of foundation pit engineering. There are two modes for the specifications of insertion depth of supporting structure in the Technical Specification for Retaining and Protection of Building Foundation Excavations (JGJ120-99 $)^{[1]}$ : one is the control over the cantilever- and single point-bearing support pile (wall) through the overturning resistant stability with the pile (wall) bottom as the fulcrum; the other is the control over cement earth-retaining wall and multiple point-bracing support line (wall) through the circular sliding stability at the bottom of supporting structure. JIANG Hong-wei (1997) ${ }^{[2]}$ obtained the anisotropic undrained shear strength through the Sekiguchi-Ohta anisotropic constitutive equation and calculated the insertion depth for anti-upheave of foundation pit by taking the last support as the circle center and through the circular sliding at the bottom of the pile (wall). In light of the reinforcement effect of soft soil at the pit bottom, HU Zhan-fei $(2001)^{[3]}$ subjected foundation soil layer togeneralizing homogeneous processing by means of weighted mean. By taking the current excavation face as the center of circular arc that goes through the bottom of supporting structure, the author applied the limit equilibrium method to analyze the stability coefficient at different insertion depth and proposed the value-taking method of insertion depth according to the different surrounding environment of foundation pit and the corresponding protection demands. On the basis of the upper limit analysis theory of plastic mechanics, ZOU Guang-dian $(2004)^{[4]}$ proceeded from the slip surface of Prandtl-Reisser solution of ultimate bearing capacity of foundation to establish the basic failure mode and introduced the Mohr-Coulomb Yielding Criteria to obtain the flow rule and calculate the harmonious velocity field of plastic zone, thus acquiring the ultimate bearing capacity of deep foundation pit base and finally the base upheaval resistance coefficient at the deep foundation pit. Based on the concept of disturbance degree and taking into consideration the disturbance degree 
changes caused by the variation of soil mass state, stress path, porosity ratio and water content, WANG Jing-chun $(2005)^{[5]}$ took the slip surface of Prandtl-Reisser solution as the sliding failure pattern of bottom soil and analyzed the influence of the changes in disturbance degree of bottom soil in the process of excavation on the security of insertion depth of supporting structure. HU Zhi-ping $(2006)^{[6]}$ simulated the process of foundation pit excavation with elastoplastic finite element program, calculating stress state of a soil element before and after foundation pit excavation(depth of different enclosure walls into earth) and limiting condition function at cell node and artificially selecting the starting and end point of the maximally possible plane of upheaval fracture at the bottom. The Dijkstra method $^{[7]}$ is employed to find the shape and location of potential plane of fracture prone to upheaval fracture in the foundation pit soil and calculate the corresponding stability coefficient at different insertion depth. WANG Hong-xin $(2007)^{[8]}$ calculated, analyzed and verified the limitations of different limit equilibrium method at different sliding surfaces on the basis of probing into the different failure types of the bottom soil. In accordance with the numerical simulation analysis method, CHENG Qi (2007) ${ }^{[9]}$ thought that the underground diaphragm wall display obvious kicking changes at the insertion ratio of 0.5 and there is a Mohr-coulomb plasticity point at the corner of wall. At the insertion ratio of 1.17 or 0.83 and with all wall toes entering hard plastic sticky soil, there is no salient difference in the maximal horizontal displacement of underground diaphragm wall and basic same form between horizontal displacement curve and measured curve.

The aforesaid literature and research achievements indicate that: (1) The analytical solution method of the insertion depth (insertion ratio) of supporting structure is solved by the ultimate balance theory of sliding stability (upper limit analysis method in literature ${ }^{[4]}$ is essentially the limit equilibrium solution of plastic zone velocity field); (2) There are two modes for the establishment of slip boundary of limit equilibrium theory: one is the sliding arc with the last cross arm pivot or current excavation face as the the circle center and the distance between circle center and wall tow; the other one is sliding surface hypothesis based on certain bearing capacity theory with the slip surface going through the wall tow; (3) When the numerical analysis method is adopted, the fracture plane with the minimal stability coefficient is calculated as the maximally possible plane of fracture. However, it is also noted that the aforesaid research achievements also have their shortcomings: (1) The solution based on limit equilibrium theory is carried out on condition of foundation soil as the homogeneous material; (2) As the "deterministic" assumption is employed for slip boundary, the result might not be the "minimum value"; (3) There is a lack of uniform evaluation criteria in numerical calculation method.

Based on the above-mentioned research results and deficiencies, this paper proposes to describe the sliding safety of foundation pit bottom with motion-unit method.

\section{"Deterministic" Model of Motion-unit Method}

\section{Unit-motion Analysis [10]}

After the objects of study are dispersed to motion units, the core of unit motion is shear plasticity sliding of moving interface. According to its relative location, the moving interface can fall into external boundary and internal boundary; if the known conditions of moving boundary are taken into consideration, it is divided into known movable boundary and unknown movable boundary. According to the fundamental assumption of motion-unit method, if a certain motion unit causes unit virtual displacement of unit boundary under the effect of certain disturbance factors, the adjacent unit will be subjected to the corresponding displacement; however, there is no relative normal displacement on the common border of random adjacent units. Therefore, the vector field of dispersed motion-unit displacement can be expressed as:

$$
\{\delta\}=\left\{u_{1}, v_{1}, u_{2}, v_{2}, \mathrm{~L}, u_{n}, v_{n}\right\}
$$


In this formula, $u_{i}$ and $v_{i}$ stand for the component of unit $i$ displacement vector in the direction of $x$ and $y$. If two random adjacent units $i$ and $j$ are taken and suppose there is a common border. between these two adjacent units, their relative displacement vectors in the direction of $x$ and $y$ are $u_{i, j}$ and $v_{i, j}$ respectively.

$$
u_{i, j}=u_{i}-u_{j}, \quad v_{i, j}=v_{i}-v_{j}
$$

Suppose $l_{\gamma}^{i}$ and $m_{\gamma}^{i}$ are the direction cosine of included angle between the exterior normal of unit $i$ on the common border $\gamma$ and the coordinate axis $x$ and $y$ and $l_{\gamma}$ and $m^{j} \gamma$ the direction cosine of included angle between the exterior normal of on the common border.and the coordinate axis $x$ and $y$. According to the assumption that the common border has no normal displacement, there is :

$$
u_{i} l_{\gamma}^{i}+u_{j} l_{\gamma}^{j}+v_{i} m_{\gamma}^{i}+v_{j} m_{\gamma}^{j}=0
$$

Given the known movable boundary $\zeta$ and the basic concept of displacement method, suppose $l^{k i_{\zeta}}$ and $m^{k i_{\zeta}}$ are the direction cosine of the included angle between the given unit displacement of unit $k$ on the known movable border $\zeta$ and the coordinate axis $x$ and $y$. If the given unit virtual displacement on the known movement trend direction is taken as the known quantity, there is:

$$
u_{k i} l_{\zeta}^{k i}+v_{k i} m_{\zeta}^{k i}=1
$$

In this formula, $u_{k i}$ and $v_{k i}$ stand for the displacement component of the displacement component of unit $k$ on the known movable boundary $\zeta$ in the direction of $x$ and $y$.

Such displacement constraint equations as formula (3) and (4) are derived from all the motion units in the assumed plastic zone discrete range according to the known movable boundary and unknown movable boundary; finally, the overall discrete motion equations are presented as:

$$
\left\lfloor K_{u v} \mid\{\delta\}+\left\{\delta^{\prime}\right\}=0\right.
$$

In which, $\left[K_{u v}\right]$ is the motion matrix of unit displacement, among which, each element is presented by the exterior normal direction cosine of unit boundary; $\{\delta\}$ is the unknown vector consisting of unit displacement to be obtained; $\left\{\delta^{\prime}\right\}$ is the constant progression vector that is obtained from zero element and virtual displacement with unit known movable boundary.

The solution of equation (5) generates the displacement of all motion units on $x$ and $y$ under the disturbance of unit virtual displacement on the known movable boundary. The relative tangential displacement along the common border $\gamma$ of two random adjacent units $i$ and $j$ is obtained from formula (6):

$$
u_{i, j}^{s}=\left(u_{i}-u_{j}\right) l_{\gamma}^{i}+\left(v_{i}-v_{j}\right) r_{\gamma}^{r_{i}}
$$

In this formula, $\dot{l}_{\gamma}^{i}$ and $\stackrel{r}{m}_{\gamma}^{i}$ are the direction cosine of the included angle between the tangent line of unit $i$ on common border $\gamma$ and the coordinate axis $\mathrm{x}$ and $\mathrm{y}$ respectively. According to the property of $u_{i, j}^{s}$, especially if its value is higher or lower than 0 , it is possible to judge the relative tangential displacement of the two adjacent units $i$ and $j$ and determine the effect direction of the frictional force that acts on the common border $\gamma$.

Unit Static Analysis

According to the principle of unit static equilibrium, the physical power that acts on random motion unit $i$ and the normal and tangential concentration on the boundary constitute the equilibrium force system:

$$
\left\{\begin{array}{l}
\sum_{\alpha=1}^{\eta} N_{\alpha x}^{i}+\sum_{\alpha=1}^{\eta} T_{\alpha x}^{i}+W_{x}^{i}=0 \\
\sum_{\alpha=1}^{\eta} N_{\alpha y}^{i}+\sum_{\alpha=1}^{\eta} T_{\alpha y}^{i}+W_{y}^{i}=0
\end{array}\right.
$$


In this formula, $N_{\alpha x}^{i}$ and $N_{\alpha y}^{i}$ stand for the component of normal force on the motion unit $i$ boundary in the direction of $x$ and $y ; T_{\alpha x}^{i}$ and $T_{\alpha y}^{i}$ represent the component of tangential force on the motion unit $i$ boundary in the direction of $x$ and $y ; W_{x}^{i}$ and $W_{y}^{i}$ stand for the component of the physical power of unit $i$ in the direction of $x$ and $y$, and $\eta$ refers to the numbers of boundary of motion unit $i$.

For the geotechnical materials, suppose that the normal stress and tangential stress on the common border $\gamma$ between random motion unit $i$ and adjacent motion unit $j$ all meet the Coulomb's shear strength principle and the exerting degree of tangential force on the shearing surface that is similar to Bishop sub-section method of slope is adopted. The tangential force $T_{\alpha}$ on the boundary surface can be expressed as ${ }^{[10 \sim 11]}$ :

$$
T_{\alpha}=\tau_{\alpha f} L_{\alpha}=\left(N_{\alpha} \tan \varphi_{\alpha}+c_{\alpha} L_{\alpha}\right) / K
$$

In this formula, $c_{\alpha}$ and $\varphi_{\alpha}$ are the shearing strength parameter of boundary rock mass; $L_{\alpha}$ is the length of common border $\gamma$ of the $\alpha^{\text {th }}$ boundary of unit $i$; $N_{\alpha}$ is the normal force of common border $\gamma$ of the $\alpha^{\text {th }}$ boundary of unit $i$; $K$ is the stability coefficient and the exerting degree of interfacial strength.

It can be seen from formula (8) that there is only one, either the tangential force or the normal force on the random boundary of motion unit, that is independent. From the perspective of problem solution, suppose $N_{\gamma}$, the normal force on the boundary, is the independent unknown variable with formula (8) substituted in (7) and equilibrium equation established for each unit of the discrete motion unit field, it is possible to obtain the equations with normal force as the unknown variable on the motion unit boundary:

$$
\left[K_{N}\right]\{N\}+\{Q\}=0
$$

In this formula, $\left[K_{N}\right]$ refers to the static force matrix of motion unit, whose elements are determined by the frictional coefficient of the boundary surface and movable boundary surface between motion units and possess the characteristic of asymmetry; $\{N\}$ is the unknown column vector consisting of normal force that acts on the boundary between motion units and movable boundary surface; $\{Q\}$ is the column vector of known normal force, which is jointly determined by the physical power of unit and binding power of boundary.

Formula (9) implies the strength development degree that is allowed by the engineering, or the stability coefficient $\mathrm{K}$, which is obtained through the iterative computations of formula (9).

\section{Chance-constrained Programming Model of Motion-unit Method}

\section{The Concept of Chance-constrained Programming}

The analytical thought of Chance-constrained programming theory ${ }^{[11 \sim 12]}$ takes into account the random variables contained in constraint conditions, allowing the safety evaluation decision made, to some extent, not to meet the constraint conditions. However, this decision should make the probability of the satisfaction of constraint condition no less than a certain confidence level $\rho$. Therefore, motion unit cannot satisfy the problem described ${ }^{[11]}$. The corresponding multi-objective chance-constrained programming model can be expressed as:

$$
\left\{\begin{array}{l}
\min \left[f_{1}(x), f_{2}(x)\right] \\
\text { s.t. } \\
\mathrm{P}_{\mathrm{r}}\left\{f_{i}(\boldsymbol{x}, \xi) \geq f_{i}^{\prime}\right\} \geq \beta_{i}, i=1,2, \mathrm{~L} \\
\mathrm{P}_{\mathrm{r}}\left\{g_{j}(x, \xi) \leq g_{j}^{\prime}\right\} \geq \rho_{j}, j=1,2, \mathrm{~L}
\end{array}\right.
$$

In this formula, $f_{i}(\boldsymbol{x}, \xi)$ is the multi-objective and $g_{i}(\boldsymbol{x}, \xi)$ the random constraint function; among which, $f_{1}(\boldsymbol{x})$ refers to the displacement function of motion unit, determined by formula $(5) ; f_{2}(\boldsymbol{x})$ refers 
to the boundary force function of motion unit, determined by formula (9); $\boldsymbol{x}$ is a n-sphere decision vector of multi-objective function $f_{i}(x, \delta)$ or random constraint function $g_{i}(x, \xi)$. For formula (5), decision-making vector is the coordinate of motion unit, namely, $\boldsymbol{x}=\left(x_{1}, y_{1}, \ldots, x_{n}, y_{n}\right)$. For formula (9), decision vector is the boundary force of motion unit, namely, $\boldsymbol{x}=\left(N^{1}{ }_{1 x}, N^{1}{ }_{1 y}, N^{1}{ }_{2 x}, N^{1}{ }_{2 y}, \ldots, N^{1}{ }_{x}\right.$, $\left.N^{1}{ }_{y}, \ldots, N_{1 x}^{i}, N_{1 y}^{i}, \ldots, N^{m}{ }_{x}, N^{m}{ }_{y}\right) . \xi$ is a random vector; $\operatorname{Pr}\{\cdot\}$ stands for the probability for the satisfaction of an event in $\{\cdot\} . \rho_{j}$ and $\beta_{i}$ are the confidence level of the $j^{\text {th }}$ and $i^{\text {th }}$ objective; $f_{i}^{\prime}$ and $g_{j}^{\prime}$ refer to the minimum value of objective function $f_{i}(\boldsymbol{x}, \boldsymbol{\delta})$ and random constraint function $g_{j}(\boldsymbol{x}, \boldsymbol{\delta})$ at the probability level of at least $\beta_{i}$ and $\rho_{j}$.

Selection of the Constraint Conditions of Objective Function

In accordance with the total stress analysis method, the random vectors in formula (10) should include: (1) $c_{i}$, the cohesion of geomaterials and $\varphi_{i}$, the internal friction angle; among which, $i=1,2, \ldots, s$ and $s$ is the number of foundation soil layer influencing the pit bottom sliding; (2) interfacial mechanical parameter of geomaterials and concrete material, adhesive strength $c_{a i}$, friction coefficient $\mu_{a i}$, among which, $i=1,2, \ldots, t ; t$ is the number of foundation soil layer influencing the pit bottom sliding and contacting the concrete material. Given the method of compensation similar to formula (8), the random vector $\xi$ is changed to $\xi^{\prime}=\left(c_{i} / K, \varphi_{i} / K, c_{a i} / K, \varphi_{a i} / K\right)$. For the displacement of motion unit, the stability state at the bottom of foundation pit, in strict sense, is that the normal displacement of motion unit is zero along the excavation face, namely, the minimum value $f^{\prime}{ }_{1}$ of objective function $f_{1}\left(\boldsymbol{x}, \xi^{\prime}\right)$ should be made $u_{i}=0$ at the probability level of at least $\beta_{1}$. For the boundary force of motion unit, the stability state at the bottom of foundation pit, in strict sense, has two representation methods:first, suppose the current operation condition is excavation state and there is no corresponding external load at the bottom surface, the minimum value $f_{2}^{\prime}$ of objective function $f_{2}\left(\boldsymbol{x}, \xi^{\prime}\right)$ should be made $N_{i \zeta}=0$ at the probability level of at least $\beta_{2}$; second, suppose there is upheaval at the pit bottom and there is a need to exert certain equilibrium external force to maintain the equilibrium state at the pit bottom, the minimum value $f_{2}^{\prime}$ of objective function $f_{2}\left(\boldsymbol{x}, \xi^{\prime}\right)$ should be made $N_{i}=P_{i}$ at the probability level of at least $\beta_{2}$. The constraint condition of objective function can be expressed as:

$$
\begin{gathered}
\mathrm{P}_{\mathrm{r}}\left\{f_{1}\left(\boldsymbol{x}, \xi^{\prime}\right) \leq 0\right\} \geq \beta_{1} \\
\mathrm{P}_{\mathrm{r}}\left\{0 \leq f_{2}\left(\boldsymbol{x}, \xi^{\prime}\right) \leq P_{i}\right\} \geq \beta_{2}
\end{gathered}
$$

If the certain slip boundary at the bottom of foundation pit is chosen and the stability coefficient obtained $K<1$, it shows that the motion units on the slip boundary are in a state of sliding instability. When the stability coefficient $K=1$, it shows that the motion units on the slip boundary are in the limit equilibrium state; when the stability coefficient $1 \leq K<F_{s}$ ( $F_{s}$ is safety factor), it means that the motion units are in a state of stability but still have not reached the safety state needed by the design; when the stability coefficient $K \geq F_{s}$, it reflects that the motion units on the slip boundary are in the state of stability and meet the safety demands.

If the bottom of foundation pit and supporting structure are regarded as the boundary of a certain unit with the designed boundary action $P_{i}$ exerted (such as the horizontal supporting force or the force of anchors of back-up coat at the bottom of foundation pit and supporting structure). If the stability coefficient calculated $K<1$, it shows that the motion unit on the slip boundary and the boundary action have already been in the state of instability of sliding; when the stability coefficient $K=1$, it indicates that after the boundary action $P_{i}$ has been exerted, the motion units on the slip boundary are in the state of limit equilibrium; when the stability coefficient $1 \leq K<F_{s}\left(F_{s}\right.$ is the safety coefficient), it means that the boundary force $P_{i}$ has already guaranteed the stability state of motion unit but failed to reach the safety state needed by the design; when the stability coefficient $K \geq F_{s}$, it indicates that under the intervention of boundary action $P_{i}$ selected, the overall state of motion unit is so stable that it can meet the safety demands.

The evaluation of probability level $\beta_{1}$ and $\beta_{2}$. With higher $\beta_{1}$, the potential sliding surface determined by $f_{1}\left(\boldsymbol{x}, \xi^{\prime}\right)$ is the most dangerous at the insertion depth of certain building envelope and 
the limiting condition of safety degree, sliding surface that has the highest confidence level; with certain $\beta_{1}$ but higher $\beta_{2}$, the boundary force needed by the most dangerous slip surface, which is determined by $f_{1}\left(\boldsymbol{x}, \xi^{\prime}\right)$ at the insertion depth of certain building envelope and limiting condition of safety degree, has the highest confidence level.

Selection of Random Constraint Function and Determination of Constraint Conditions

Researches have shown that ${ }^{[14 \sim 15]}$, the stability of random constraint functions comes in two aspects: first, the slip boundary model is taken as the constraint function; second, the medium mechanics parameter of sliding surface is taken as the random variable and a certain form of limit equilibrium theory as the constraint function. For easier comparison, the former is defined as the deterministic type ${ }^{[1 \sim 9]}$ and the latter the random variable.

The random constrain function $g_{i}(\boldsymbol{x}, \xi)$ has three forms ${ }^{[13]}$. When the medium mechanics parameters of sliding surface is seen as the random variable, the polynomial can be selected as the random constraint function, which is expressed by the Coulomb law ${ }^{[1415]}$. With the mean value of shear strength parameter in the direct shearing test of soil as random vector, the probability and statistics theory is employed to define the ratio of a sample value of shear strength parameter in the direct shearing test against other mean values as ratio of average $\lambda_{i}=a_{i} / E\{a\}$; among which, $a_{i}$ refers to any of the sample value of shear strength parameter in the direct shearing test and $E\{a\}$ the corresponding sample average. As there is marked difference in drainage function between direct shearing test and triaxial shear test, the shear strength of triaxial shear test is seen as the true value. Therefore, the mean ratio of direct shear test $\lambda_{i}$ and the shear strength of triaxial shear test $\tau_{u i}$ are regarded as the random vector $\xi$, then the random constrain condition is:

$$
\mathrm{P}_{\mathrm{r}}\left\{\lambda_{c i} c_{i}+\sigma_{i} \lambda_{\varphi i} \tan \varphi_{i} \leq \tau_{u i}\right\} \geq \rho_{i}
$$

In this formula, $\lambda_{c i}$ and $\lambda_{\varphi i}$ are the mean ratio of direct shear test $c_{i}$ and $\varphi_{i} \sigma_{i}$ is the normal stress on the sliding surface under the condition of a certain sliding surface model; $\rho_{i}$ is the probability level of the existence of random constraint condition. The value of probability level $\rho_{i}$ reflects the trust degree of calculator in the shear strength of triaxial shear test. As the stipulation of literature [1] suggests, $\rho_{i}$ has a higher value.

\section{Solution of Chance-constrained Programming Model}

\section{Equivalent Forms of Chance Constraint}

According to the solving approach ${ }^{[13 \sim 14]}$ of chance-constrained programming, the random variable contained in chance constraint is thought to meet a certain random model and converted to its equivalent form for solution. For the random constraint conditional expression (12) selected, suppose the $c_{i}, \varphi_{i}$ and $\tau_{u i}$ are mutually independent normal random variables, there is the function

$$
y\left(\xi_{i}\right)=\lambda_{c i} c_{i}+\sigma_{i} \lambda_{\varphi i} \tan \varphi_{i}-\tau_{u i}
$$

Which is also the normal random variable. At the same time, there is

$$
\begin{aligned}
& E\left(y\left(\xi_{i}\right)\right)=\lambda_{c i} E\left(c_{i}\right)+\sigma_{i} \lambda_{\varphi i} E\left(\tan \varphi_{i}\right)-E\left(\tau_{u i}\right) \\
& V\left(y\left(\xi_{i}\right)\right)=\lambda_{c i}^{2} V\left(c_{i}\right)+\sigma_{i}^{2} \lambda_{\varphi i}^{2} V\left(\tan \varphi_{i}\right)+V\left(\tau_{u i}\right)
\end{aligned}
$$

In this formula, $E(\cdot)$ and $V(\cdot)$ stand for expected deviation and variance respectively.

As the function $y(\xi)$ is the normal random variable, the chance constraint formula (12) after its standardization is equivalent to

$$
\mathrm{P}_{\mathrm{r}}\left\{\eta_{i} \leq-E\left(y\left(\xi_{i}\right)\right) / \sqrt{V\left(y\left(\xi_{i}\right)\right)}\right\} \geq \rho_{i}
$$

In this formula, $\eta_{i}$ is the standard normal distribution function and formula (13) stands. If and only if $\Phi^{-1}\left(\rho_{i}\right) \leq-E\left(y\left(\xi_{i}\right)\right) / \sqrt{V\left(y\left(\xi_{i}\right)\right)}$ 
There is the equivalent form of random constraint condition formula (12)

$$
\begin{aligned}
& \lambda_{c i} E\left(c_{i}\right)+\sigma_{i} \lambda_{\varphi i} E\left(\tan \varphi_{i}\right)+ \\
& \Phi^{-1}\left(\rho_{i}\right) \sqrt{\lambda_{c i}^{2} V\left(c_{i}\right)+\sigma_{i}^{2} \lambda_{\varphi i}^{2} V\left(\tan \varphi_{i}\right)+V\left(\tau_{u i}\right)} \leq E
\end{aligned}
$$

Computing Method

In accordance with the aforesaid chance constraint analysis method of motion unit, the genetic algorithm contains the following computing steps:

(1) determine the potential sliding surface, subject the motion field to mesh discretization and mark out $\mathrm{n}$ motion units according to the determination method of a certain sliding surface;

(2) apply the virtual disturbance displacement $\Delta_{i}$ of certain forms to a motion unit and adopt formula (5) to calculate the unit displacement of motion unit field;

(3) employ formula (9) to calculate normal force $N_{\alpha i}$ and tangential force $T_{\alpha i}$ on the unit interface;

(4) randomly generate random variables according to the probability distribution of parameter, test the feasibility of chance constraint formula (12) and take it as the initial chromosome in the genetic process;

(5) subject chromosomes to cross-footing and variation calculation and test the feasibility of its offspring with formula (12);

(6) rotate the roulette to select chromosome until the circulation coefficient that can meet the stability coefficient iteration is found.

Table 1 normal distribution of mechanics parameter of soil

\begin{tabular}{|c|c|c|c|c|c|}
\hline Formation name & depth $(\mathrm{m})$ & Severity $\left(\mathrm{kN} / \mathrm{m}^{3}\right)$ & $\begin{array}{c}\text { Modulus of } \\
\text { Compression }(\mathrm{MPa})\end{array}$ & Cohesive Force $(\mathrm{kPa})$ & $\begin{array}{c}\text { Internal Friction } \\
\text { Angle }\left({ }^{\circ}\right)\end{array}$ \\
\hline miscellaneous fill & 2.6 & $N(18.6,1.32)$ & $N(8,0.25)$ & $N(5,0.02)$ & $N(22,1.96)$ \\
\hline silty clay & 4.7 & $N(19.7,0.15)$ & $N(8,0.03)$ & $N(32.5,0.5) 4$ & $N(16,0.20)$ \\
\hline clay & 11.2 & $N(19.30, .25)$ & $N(8.67,0.17)$ & $N(37.5,0.5) 7$ & $N(20,0.78)$ \\
\hline clay & 15.6 & $N(18.5,0.16)$ & $N(9.28,0.07)$ & $N(35,0.61)$ & $N(21.5,0.25)$ \\
\hline limestone & --- & $N(26.8,0.02)$ & $N\left(2.25 \times 10^{4}, 10.95\right)$ & $N\left(1.45 \times 10^{3}, 22.46\right)$ & $N(47.5,0.48)$ \\
\hline
\end{tabular}

Table 2 normal distribution parameter of average value radio

\begin{tabular}{|cc|c|c|c|}
\hline & Computation Parameter & $\lambda_{c}$ & $\lambda_{\varphi}$ & $\tau_{u}$ \\
\hline Formation name & & $N(0.68,0.09)$ & $N(0.63,0.07)$ & $N(29.2,0.63)$ \\
\hline $1(1)$ & miscellaneous fill & $N(0.79,0.04)$ & $N(0.84,0.04)$ & $N(35.7,0.79)$ \\
\hline (2) & silty clay & $N(0.82,0.05)$ & $N(0.77,0.06)$ & $N(43.5,0.82)$ \\
\hline (3) & clay & $N(0.87,0.07)$ & $N(0.82,0.09)$ & $N(40.9,0.91)$ \\
\hline (4) & clay &
\end{tabular}

\section{Computational Analysis of Examples}

\section{Calculation Parameters and Calculation Model}

Take the subway station as an example. The foundation pit of station has an excavation depth of $18.4 \mathrm{~m}$ and width of $18.7 \mathrm{~m}$. The cast-in-situ bored pile building envelope with the clear spacing of $0.6 \mathrm{~m}$ and diameter of $0.8 \mathrm{~m}$ is adopted with the lateral bracing set at the excavation depth of $1.0 \mathrm{~m}$, $8.6 \mathrm{~m}$ and $15.6 \mathrm{~m}$. In light of the construction load on the ground outside pit, $q=30 \mathrm{kN} / \mathrm{m}$. Standard strength partial factor $\gamma_{k}=1.3$ is taken as the strength reduction factor $F_{s}$. Suppose that the physical mechanical property of the field foundation is conformed to normal distribution, Table 1 lists the normal distribution parameter $N\left(\chi, \sigma^{2}\right)$ of the soil of field foundation, in which, $\chi$ is the mean value and $\sigma^{2}$ is the variance. Table 2 presents the normal distribution parameter of $\left(\lambda_{c i}, \lambda_{\varphi i}\right)$, the average value ratio of shear strength parameter after reduction, and the triaxial shear strength $\tau_{u i}$ (due to the small frictional angle in the triaxial shear test, the triaxial shear strength is taken as $\left.\tau_{v i}=C_{u i}\right)$. In formula 
(12), the probability level $\beta_{\text {ind }}$ and are made $95 \%$. The crossover probability $P_{c}$ and mutation probability $P_{m}$ of genetic computation are 0.28 and 0.18 respectively.

Two computation modes are employed for comparison. The first is the smooth sliding method ${ }^{[1]}$ (Fig. 1). Suppose that the last supporting point $O$ is the fixed point, then the $O$ is the circle center of slip circle. In fact, the slip circle center might exist at a certain place ${ }^{[5 \sim 6]}$ between the last supporting point $O$ and pit bottom $O$ ', which is expressed as $\xi$ with $0 \leq \xi \leq 2.8 \mathrm{~m}$. First, the position of shear opening $\mathrm{A}$ on the sliding surface at the pit bottom is determined according to the arc center and controlled by the foundation pit width $B_{0}$, the possible sliding arc radius should be $R=\xi+D_{p} \leq B_{0}$ after the sliding center has been chosen. The second is the slip surface of Prandtl-Reisser Solution ${ }^{[4]}$ (Fig. 2 ). The position of shearing opening $A$ on the sliding surface at the pit bottom is determined by $L_{S}^{[10 \sim 11]}$

$$
\left\{\begin{array}{l}
L_{\min s} \leq L_{s} \leq 2 L_{\min s} \\
L_{\min s}=r_{0} \exp [\pi \tan (\varphi / 2)] \cos \left(45^{\circ}-\varphi / 2\right) \\
r_{0} \leq b_{0} / 2 \cos \left(45^{\circ}+\varphi / 2\right)
\end{array}\right.
$$

\section{Computational Analysis}

For the method of circular sliding surface, the sliding arc central angle $\triangle \mathrm{AOC}$ is divided into 6 equal parts. BC and CD, the Prandtl-Reisser log spiral, are equally divided into three sections and each circular sliding surface or log spiral slip surface all simplified into straight lines, thus setting up the motion unit field. The method of circular sliding surface generates different $\xi$ value at different insertion ratio. In Prandtl-Reisser method, the $O^{\prime}$ point $(\xi=0)$ at the pit bottom is taken as the rotation center of sliding soil mass. Therefore, the maximal insertion ratio is 0.74 for the aforesaid foundation pit.

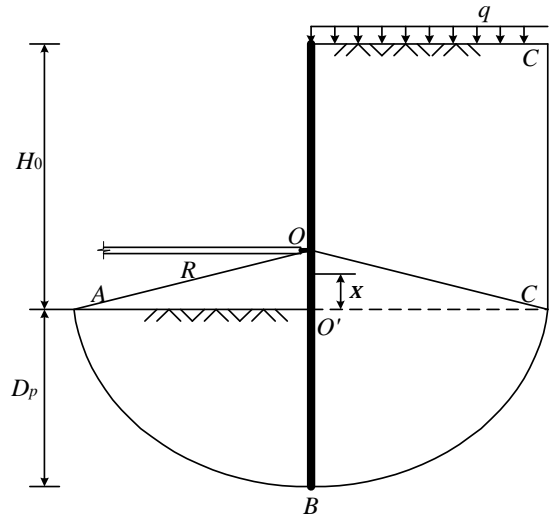

Fig.1 Module of circular sliding surface

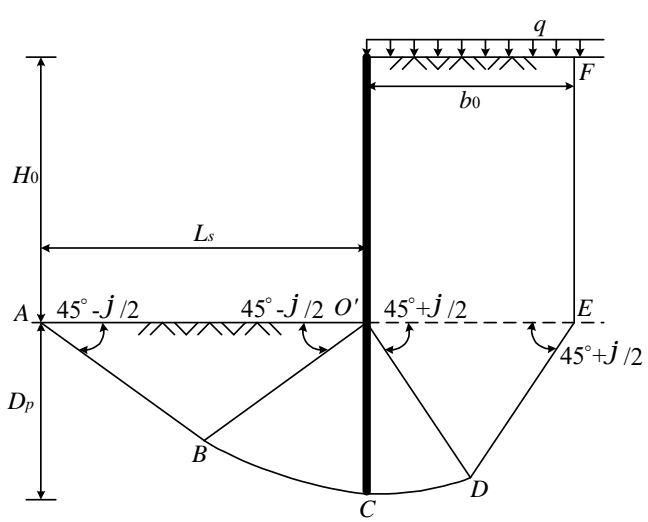

Fig.2 Module of Prandtl' sliding surface

If formula (11a) is taken as the constraint condition of objective function, the relationship between the insertion ratio of circular slipping method and stability coefficient, as shown in Fig. 3, can be obtained. Similar to the rules of literature ${ }^{[8]}$, the stability coefficient displays non-linear decreasing increase as $\xi$ reduces at the insertion ratio of less than 0.6 . The difference is that at the insertion ratio of more than 0.6 , with $\xi$ increasing, the stability coefficient transforms from parabolic growth to approximate linear growth. Besides, at the same insertion ratio, the lower $\xi$ is usually accompanied by higher stability coefficient because despite the same insertion ratio, the lower $\xi$ usually gives rise to the smaller volume of sliding soil mass and smaller soil mass load on $O^{\prime} C$ surface. Fig. 4 presents the relationship between the failure probability of circular slide and the location of circle center. Therefore, it can be seen that: (1) regardless of the insertion ratio, higher position of the circle center, or higher $\xi$, is accompanied by higher failure probability; (2) as the insertion ratio increases and the position of circle center rises ( $\xi$ increases), the decline degree of failure probability gradually tapers. Similarly, the comparison of circular slip method with $\xi=0$ and the relationship between insertion ratio of Prandtl-Reisser method and stability coefficient is shown in Fig. 5 and the the comparison 
with failure probability in Fig. 6. It is seen from Fig. 5 that under the condition of $\xi=0$, the stability coefficient of two slip surfaces takes on completely different forms because with increasing insertion ratio, the limit sliding soil mass volume of circular slip method is mainly evidenced by the increasing depth. For this reason, the limit sliding soil mass volume of Prandtl-Reisser method is demonstrated by the increasing width of foundation pit bottom, which makes the rapid growth of stability coefficient tend to the limit and results in the approximate hyperbolic-type. ( It also can be seen from Fig. 6) that the stability failure probability of circular slip method is higher than that of method, which is associated with the volume of the sliding soil mass behind the wall of two types of slip surface. The volume of sliding soil mass behind the wall of circular slip method is significantly higher than that of Prandtl-Reisser method.

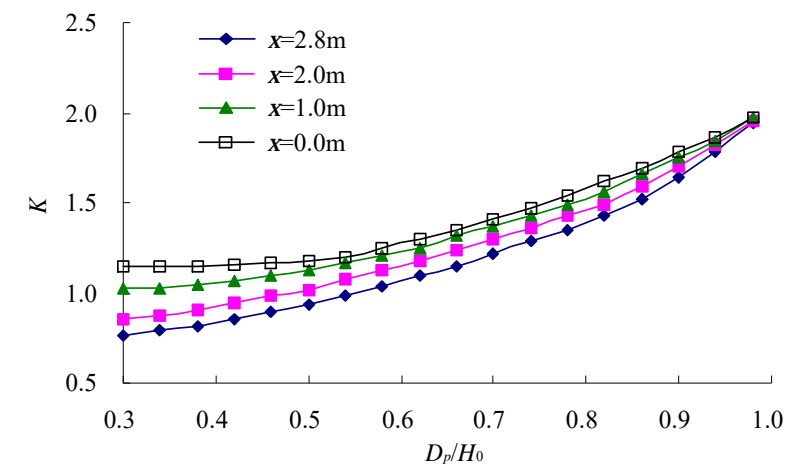

Fig.3 Relationship between insertion ratio and stability factor of circular sliding surface

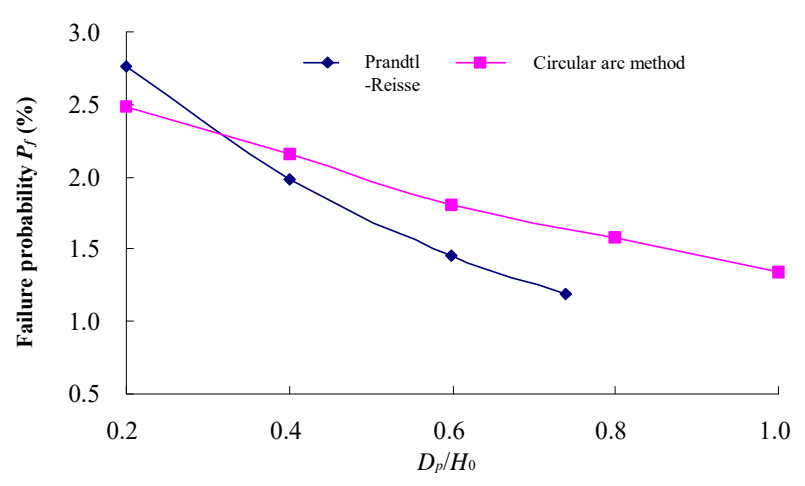

Fig.5 Comparison of relationship between insertion ratio and stability factor of two sliding surface modes

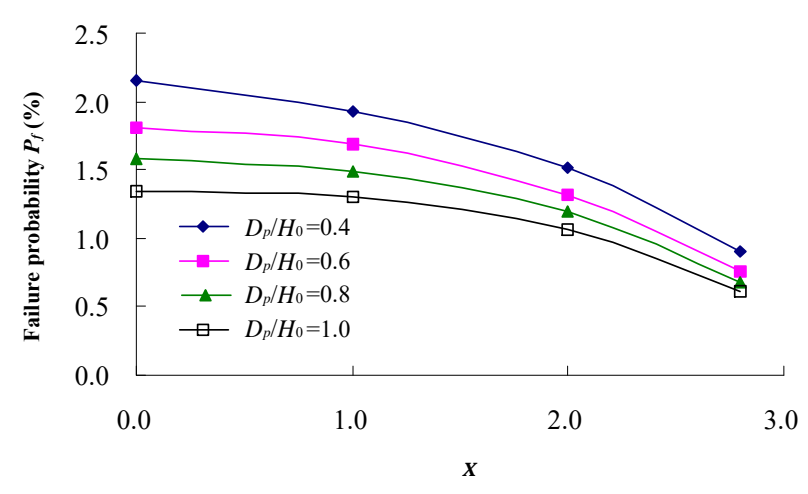

Fig.4 Relationship between the centre of a circle and failure probability

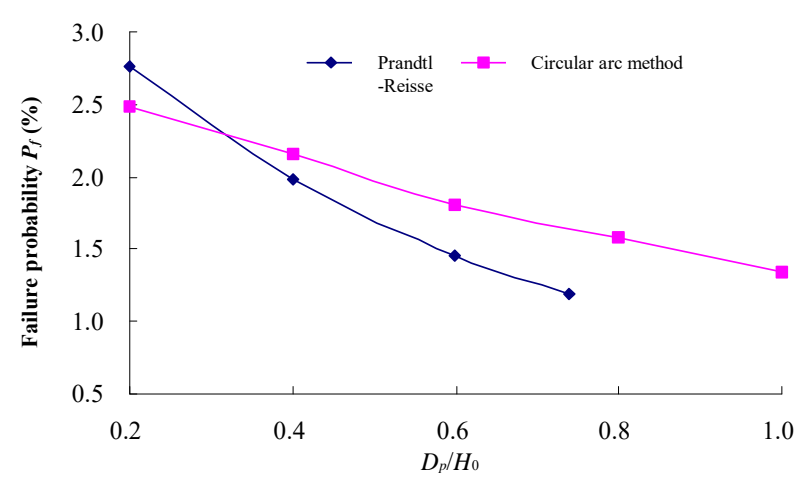

Fig.6 Comparison of relationship between insertion ratio and failure probability of two different sliding surface modes

There are two modes if formula (11b) is taken as the constraint condition of objective function. In the first mode, the distributed load $P_{i}$ is thought to be at the pit bottom (similar to the back-pressure load $P_{i}$ when the foundation pit bottom reaches the limit equilibrium state). Fig. 7 and 8 respectively demonstrate the computation results of circular slip method (when $\xi=2.8 \mathrm{~m}$ ) and Prandtl-Reisser method (when $\xi=0.0 \mathrm{~m}$ ) with distributed load at the pit bottom. Fig. 7 reveals that at the insertion ratio 


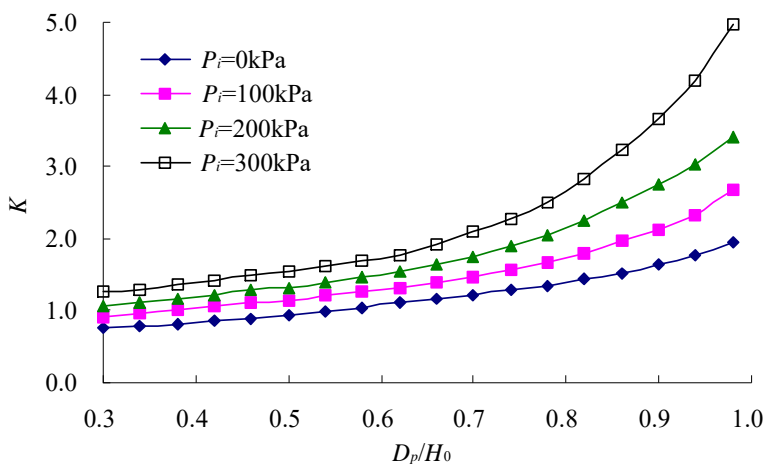

Fig.7 Influence of different pit bottom stack load on stability coefficient based on circular sliding method

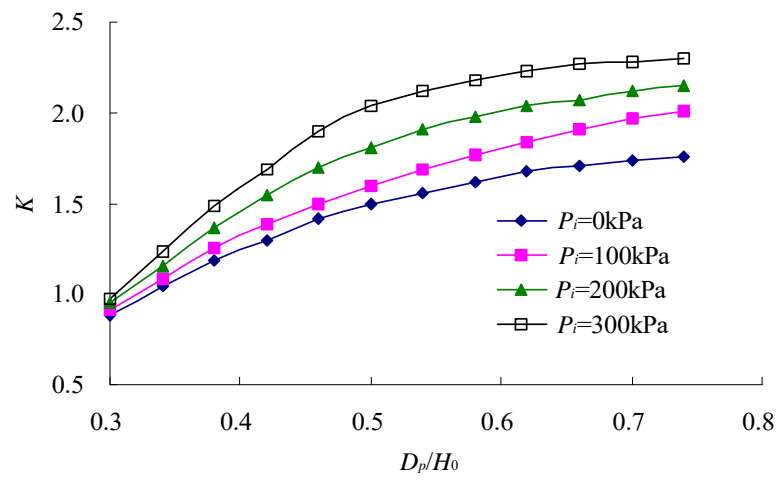

Fig.8 Influence of different pit bottom stack load on stability coefficient based on Prandtl-Reisser method

of more than 0.65 , the circular slip method can markedly enhances its stability coefficient along with the increase in back-pressure load at the pit bottom; however, at the insertion ratio of less than 0.65 , the increase in stability coefficient is basically in linear proportion to the increase in back-pressure load. However, in Prandtl-Reisser method, as the back-pressure load increases, the stability coefficient tends to an asymptotic value.

In the second mode, in light of the lateral load on the supporting structure (similar to the interior support of the foundation pit or external support of anchor stock), the influence of lateral load of circular slip method (when $\xi=0.0 \mathrm{~m}$ ) is presented in Fig. 9. It can be seen from Fig. 9 that for the lateral supporting force of less than $800 \mathrm{kN}$, the increase of stability coefficient transfers from the parabolic type to approximate linear type; however, for the lateral supporting force of more than $800 \mathrm{kN}$, the increase of stability coefficient gradually transforms to the approximate hyperbolic model. At the insertion ratio of lower than 0.6 , the growth rate of stability coefficient takes on proportional increase along with the horizontal supporting force. At the insertion ratio o higher than 0.6 , the growth rate of stability coefficient significantly reduces. At the horizontal supporting force of $1600 \mathrm{kN}$ and insertion ratio of more than 0.8 , the stability coefficient basically remains unchanged. It can be speculated from the mechanism of horizontal supporting face in enhancing the stability of pit bottom that the horizontal supporting force that is far away from the pit bottom is less effective in increasing the stability coefficient of pit bottom. Therefore, Fig. 10 offers a comparison of the two slip methods at the horizontal supporting load of $200 \mathrm{kN}, 500 \mathrm{kN}$ and $800 \mathrm{kN}$ (Fig. 10).

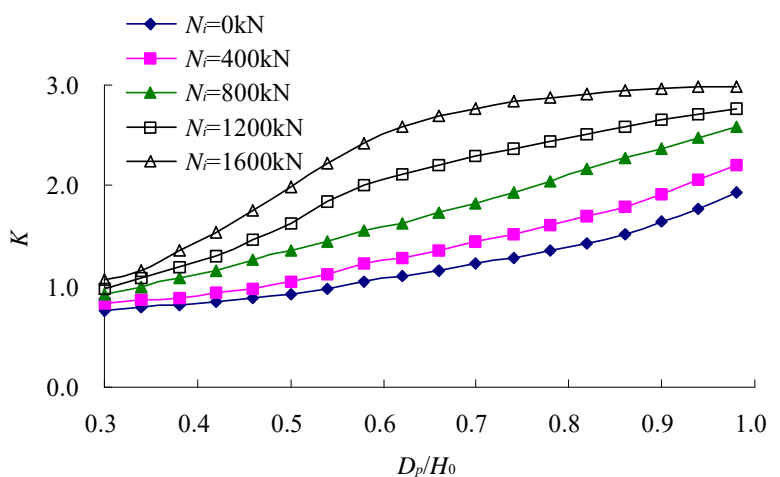

Fig.9 Influence of final bracing load based on circular sliding method on stability coefficient

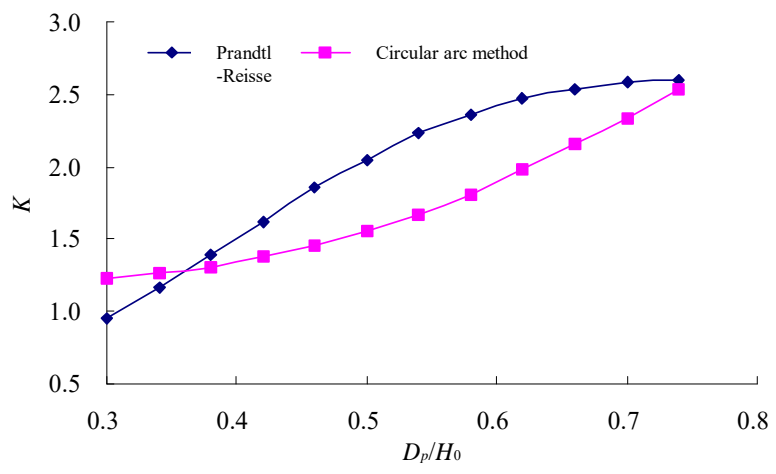

Fig.10 Relationship between insertion ratio and stability coefficient of bracing load for difference sliding surface 


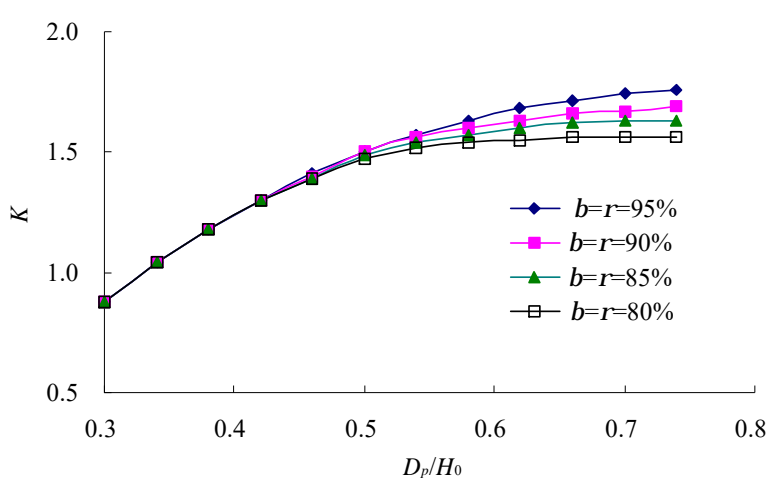

Fig.11 Influence of value on the relationship between insertion ratio of Prandtl' sliding surface stability factor

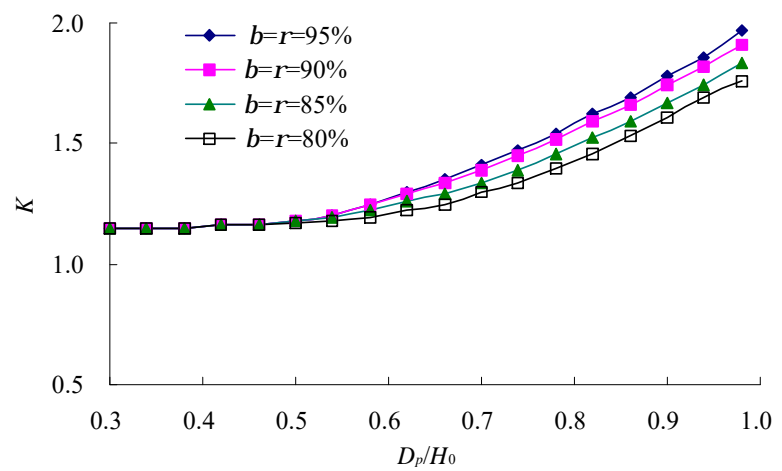

Fig.12 Influence of value on the relationship between insertion ratio of circular sliding surface and stability factor

$\beta$ and $\rho$ objective, the objective function of chance-restrained programming and the confidence level of random constraint function values respectively, are theoretically the higher, the better. However, in reality, higher confidence level also requires high costs. Fig. 11 and 12 present the relationship between the insertion ratio and stability coefficient at different evaluation of $\beta$ and $\rho$. The circular slide method $(\xi=0.0 \mathrm{~m})$ and Prandtl-Reisser method slip surface basically take the insertion ratio of $0.42 \sim 0.50$ as the demarcation point. When the $D_{p} / H_{0}$ is lower than the demarcation point, the changes of $\beta$ and $\rho$ basically has no impact on the stability coefficient of different insertion ratio. However, when the $D_{p} / H_{0}$ is higher than the demarcation ratio, the gradual decrease in $\beta$ and $\rho$ reduces the stability coefficient as the stability coefficient descends. The demarcation point of insertion ratio can be understood as: (1) at the insertion ratio of less than 0.42 or 0.50 , the decrease in confidence level $\beta$ and $\rho$ will not change the stability of pit bottom. The unsafe insertion ratio refers to the chaotic characteristics of foundation soil with no possibility of the stability state at the pit bottom; (2) For the insertion ratio of higher than 0.42 or 0.50 , the decrease in confidence level $\beta$ and $\rho$ will decrease the stability at the pit bottom; in other words, the safety of appropriate insertion ratio is mainly determined by the credibility of the property of foundation soil.

\section{Conclusion}

The establishment of the above-mentioned model and computational analysis of examples generate the following conclusions:

(1) The motion unit method can be employed to establish the deterministic model for the analysis of bottom stability of foundation pit, which is placed at certain confidence level with the property of foundation soil of foundation pit and boundary load (stack load at the pit bottom, bracing load, stack load on the ground outside the pit) as the random constraint function. In this case, the model for the analysis of chance-constrained programming of motion-unit method is established, which can be applied to the possible sliding forms at the pit bottom;

(2) For the circular sliding method, the position of sliding circular center can significantly influence the pit bottom stability at different insertion ratio. The higher position of sliding circular center ( $\xi$ is higher), regardless of the insertion ratio, is accompanied by the marked decrease of failure probability;

(3) For the two slip surfaces, Prandtl-Reisser method is accompanied by higher possibility of wedge sliding object at the pit bottom. At higher insertion ratio, the circular sliding has higher failure probability;

(4) Lateral bracing is conducive to the stability of pit bottom soil. The last wailing effect is so crucial that it can double the stability coefficient of pit bottom;

(5) At the insertion ratio of less than 0.42 or 0.50 , the confidence of random constraint function will basically not influence the pit bottom stability. 


\section{References}

[1] JGJ120-99, Technical specification for retaining and protection of building foundation excavations[S]. Beijing: China Architecture and Building Press, 1999. (in Chinese)

[2] JIANG Hong-wei, ZHAO Xi-hong, ZHANG Bao-lian. An analysis of heave-resistant stability of deep foundation pit in weak clay under anisotropic conditions[J]. Chinese Journal of Geotechnical Engineering, 1997, 19(1):1 7.(in Chinese)

[3] HU Zhan-fei, ZHOU Jian, YANG Lin-de. Study on the stability of the weak soil at the bottom of deep foundation pit[J]. China Civil Engineering Journal, 2001, 34(2):84 88, 95. (in Chinese)

[4] ZOU Guang-dian. An analysis of heave-resistant stability of deep foundation pit based on an upper limit analysis method[J]. Rock and Soil Mechanics,2004,25(12):1873 1878(in Chinese)

[5] WANG Jing-chun, XU Ri-qing, HUANG Bin. Study on heave-resistant stability of deep foundation pit under the construction disturbance[J]. Chinese Journal of Rock Mechanics and Engineering, 2005, 24(Supp.2):5 405-5 409. (in Chinese)

[6] HU Zhi-ping, YAO Hai-ming, LUO Lin-juan. Study on the combination optimization method based on Dijkstra algorithm in searching potential upheaval fracture plane of foundation pit[J]. Rock and Soil Mechanics, 2006, 27(9):1583 15878. (in Chinese)

[7] Feng Z L, Lewis R W. Optimal estimation of in-situ ground stresses from displacement measurements[J]. International Journal for Numerical and Analytical Methods in Geomechanics, 1987, 11(6): 391-408.

[8] WANG Hong-xin, CHEN Jian-jun, LIU Ji-shan. Practical calculation analysis and application of safety factor of upheaval-resistant stability of foundation pit[J]. Chinese Journal of Rock Mechanics and Engineering, 2007, 26(s1): 3223 3230. (in Chinese)

[9] CHENG Qi, LIU Guo-bin, ZHANG Wei-li. Finite element analysis of deformation of Subway ultra-deep and deep foundation pit in Shanghai[J]. Chinese Journal of Underground Space and Engineering, 2009, 5(s2):1498 1502. (in Chinese)

[10] LUO Xiao-hui. Determination of bearing capacity of foundation soil with genetic algorithm[J]. Chinese Journal of Rock Mechanics and Engineering, 2001, 20(3): 394 398. (in Chinese)

[11] LIN Yu-liang. Uncertainty Problem in geotechnical and structural engineering and the analysis methods[M]. Beijing, Science Press, 2009, 395 399. (in Chinese)

[12] Iwamura K., Liu B. A genetic Algorithm for Chance Constrained Programming[J]. Journal of Information \& Optimization Science. 1996, 17(2): 40-47.

[13] LIU Bao-xuan, ZHAO Rui-qing. Stochastic programming and fuzzy programming[M]. Beijing: Tsinghua University Press, 1998.(in Chinese)

[14] LUO Xiao-hui, LI Zai-guang, HE Li-hong.Analysis method of chance constrained programming with the protection of soil nail[J]. Engineering Mechanics, 2007, 24(12):128 134.

[15] LUO Xiao-hui, HE Li-hong.Chance constrained method for the programming of slope stability[J]. Chinese Journal of Rock Mechanics and Engineering, 2008, 27(s1): 2821 2824. (in Chinese) 